\title{
On the validity of simple precipitate size measurements by small-angle scattering in metallic systems
}

\author{
Alexis Deschamps and Frédéric De Geuser \\ SIMAP, Grenoble INP - CNRS - UJF, 1130 rue de la Piscine, BP 75, Saint Martin d'Hères Cedex, \\ 38402, France. Correspondence e-mail: alexis.deschamps@grenoble-inp.fr
}

\begin{abstract}
This paper assesses how simple small-angle scattering particle size evaluation models, such as Porod or Guinier radii, which have a normally limited validity range, may see this range extended to larger $q$ values. This is shown to be particularly true for metallic systems, where the dispersion in particle size is always large. Because of the size dispersion, the relationship between the average particle size and the Guinier radius is shown to change. For systems with relatively large size dispersion, the paper shows that the Porod and Guinier radii, and simple extensions thereof, give valuable information on particle size and particle size distribution. This is demonstrated to be valid for particles with moderate aspect ratios. These simple evaluations are quick and very well adapted to large data sets, such as those originating from time-resolved or scanning small-angle experiments.
\end{abstract}

\section{Introduction}

Small-angle scattering (SAS) is one of the most common techniques for measuring precipitate sizes and precipitate size distributions in metallic systems. Carried out with X-rays or neutrons, it is classically used in aluminium alloys (Marlaud et al., 2010a; Dupasquier et al., 2007; Tsao et al., 2006), steels (Bischof et al., 2005; Michaud et al., 2007; Ohnuma et al., 2009; Perrard et al., 2006), magnesium alloys (Vogel et al., 2003; Antion et al., 2007), Ni-Ti alloys (Kompatscher et al., 2002) etc. A common and important characteristic of precipitates in metallic systems is that their size distribution is always polydisperse, and this needs to be taken into account when analysing the data.

When a SAS spectrum is recorded, several strategies can be adopted to extract useful information on the particle sizes. Algorithms have been developed to determine a particle size distribution from the scattering spectrum without any particular assumption of its form, using Fourier transforms. The indirect Fourier transform method developed by Glatter (Glatter, 1977; Glatter, 1980; Glatter \& Kratky, 1982) has been particularly well used in the literature (Leitner et al., 2007; Sato et al., 2009; Staron \& Kampmann, 2000; Ulbricht et al., 2007; Van Dijk et al., 2002; Bergner et al., 2009; Bischof et al., 2005). However, this task can be quite complicated in practice. Firstly, in the case of dilute systems with relatively weak contrast, the scattering at very small angles is often perturbed by other contributions, coming from large particles present in the sample, dislocations, sample surfaces etc. [see e.g. Kompatscher et al. (2002); Mukherji et al. (2003)]. Scattering at very small angles can also be perturbed by multiple scattering, particularly when short-wavelength X-rays are used. Secondly, counting statistics at high angles do not always permit accurate measurements, especially when fast time-resolved experiments (with low counting times) are carried out. These imperfections in the experimental intensity at the boundaries of the scattering-vector range make algorithms based on Fourier transforms difficult to use. In addition, these algorithms are generally not able to extract simultaneously the precipitate form factor and the precipitate size distribution. However, most metallurgical samples simultaneously show non-spherical particles and particle dispersion.

To overcome these difficulties, two alternative strategies can be adopted. The first is to model SAS data by calculating the scattering of a given precipitate size distribution, assuming a shape for the precipitates. The scattering by simple objects such as spheres or ellipsoids can be exactly calculated and, in a low volume fraction hypothesis, the intensity scattered by the collection of particles can be simply computed. Most commonly a lognormal distribution of particles is assumed, since it corresponds rather well with commonly observed precipitate size distributions (Alinger et al., 2009; Del Genovese et al., 2005; Glade et al., 2005; Staron et al., 2003; Vogel et al., 2003; Wiskel et al., 2008; Perrard et al., 2006; Marlaud et al., 2010b). Other types of distributions have been used less commonly as well, such as Gaussian (Michaud et al., 2007) or Shultz (Kusy et al., 2004), and one can find instances where several distributions are combined to describe the scattering by several precipitate families of different mean sizes (Bischof et al., 2008; Leitner et al., 2005, 2006; Ohnuma et al., 2009).

The second possibility is to carry out a simple evaluation directly from the scattering curve, which gives a particle size directly. Such measurements are frequently used in the 
literature and they are particularly interesting when a very large amount of data needs to be processed automatically. This situation occurs frequently now with the improvement in $\mathrm{X}$-ray detectors and brightness of synchrotron sources, which enable generalized time-resolved measurements of precipitate microstructures (Dupasquier et al., 2007; Schober et al., 2010; Kenesei et al., 2006; Deschamps et al., 2005; Marlaud et al., 2008 ) or precipitate mapping of heterogeneous microstructures (Dumont et al., 2006; De Geuser et al., 2010). In such cases, where up to several thousand SAS spectra need to be analysed, complicated parameter-dependent data analysis can be impractical or even impossible.

The most classical parameter that is extracted directly from SAS data is the Guinier radius $R_{\mathrm{g}}$ (or radius of gyration) (Kenesei et al., 2006; Kompatscher et al., 2002; Ohnuma et al., 2000; Wiskel et al., 2008; Dupasquier et al., 2007; Kamiyama et al., 2001; Deschamps et al., 2001), for which the intensity $I(q)$ is shown to be equivalent to a Gaussian for small scattering vectors $q[q=(4 \pi / \lambda) \sin \theta$, where $\theta$ is half the scattering angle and $\lambda$ is the wavelength of the incident radiation] (Guinier $e t$ al., 1956; Kostorz, 1996):

$$
I(q) \propto \exp \left(-R_{\mathrm{g}}^{2} q^{2} / 3\right) .
$$

It is well known that, in the case of a monodisperse distribution of spheres, the following relationship holds between the Guinier radius and the radius of the spheres:

$$
R_{\mathrm{g}}^{\text {monodisperse }}=(3 / 5)^{1 / 2} R_{\text {sphere }} .
$$

The Guinier approximation is valid for a particle of any shape at sufficiently small scattering vectors, where $q R<<1$. Therefore, it is generally considered that the measurement of precipitate size using this approximation should be made at the smallest scattering vectors measured during the experiment. In practice, however, either researchers do not provide the $q$ range used for the Guinier size measurement (Dupasquier et al., 2007; Kompatscher et al., 2002) or they find that the Guinier approximation is valid at larger scattering vectors, where $q R$ lies somewhere between 1 and 2 (Kenesei et al., 2006; Dumont et al., 2006). Thus, it is not clear (i) under what conditions the Guinier approximation is valid in the case of a polydisperse collection of particles; (ii) in what $q$ range it should be applied; (iii) what the relationship is between the Guinier radius and the average radius in the case of a polydisperse distribution of precipitates; and (iv) what the influence is of precipitate shape on these relationships.

The Guinier radius can be extracted from a so-called Guinier plot, where $\log (I)$ is plotted versus $q^{2}$. The Guinier radius is then simply related to the initial slope $\alpha$ of the plot by

$$
R_{\mathrm{g}}=(-3 \alpha)^{1 / 2} .
$$

If the Guinier approximation holds over a sufficiently wide range of $q$, the intensity exhibits a linear part and it can be shown that the Guinier radius can also be obtained very simply from the scattering vector $q_{\max }$ at which $I q^{2}$ shows a maximum:

$$
R_{\mathrm{g}}=R_{\max }=3^{1 / 2} / q_{\max } .
$$

Thus, the precipitate size can clearly be visualized on a Kratky plot $\left(I q^{2}\right.$ versus $\left.q\right)$ as being inversely proportional to the $q$ value at the maximum (while the volume fraction is proportional to the area of the plot). Even in those samples where the Guinier plot does not show a straight line, one can generally observe a maximum in the Kratky plot. Equation (4) applied to this maximum has been used in the past as a measurement of precipitate sizes and the so-called 'pseudo-Guinier radius' $R_{\max }$ (Perrard et al., 2006). The validity of the use of this parameter to characterize a precipitate microstructure is also unclear. The position of this maximum in the $I q^{2}$ curve can be seen as the $q$ value that contributes the most to the total integrated intensity, but its relationship to the Guinier radius may not be straightforward.

In the present paper, we will numerically calculate the SAS behaviour of lognormal distributions of precipitate sizes as a function of the width of the particle size distribution (PSD). This will be done first for spherical particles and subsequently for ellipsoids of variable aspect ratio. The SAS behaviour will be evaluated particularly in the light of studying the relationship between the measured Guinier or pseudo-Guinier radii and the actual average precipitate sizes. These values will also be compared with the Porod radius, which provides a measurement of the surface-to-volume ratio of the particles. Finally, this analysis will be matched with experimental data on the $\mathrm{Fe}-\mathrm{Cu}$ system (which presents dilute distributions of spherical precipitates) and the $\mathrm{Fe}-\mathrm{Nb}-\mathrm{C}$ system (where precipitates have an ellipsoidal shape of moderate aspect ratio). The paper will not address the case of precipitates of very large aspect ratio such as fine needles or platelets, which should be analysed using specific procedures. Similarly, we will not address the case of concentrated distributions of particles, where interference between particles makes the data analysis more complicated.

\section{SAS by a lognormal distribution of spheres}

Let us consider a distribution of spheres, the number density of which follows a lognormal distribution defined by two parameters, the median size $R_{\mathrm{m}}$ and the dispersion parameter $s:$

$$
f(R)=\frac{1}{(2 \pi)^{1 / 2} s R} \exp \left\{-\frac{1}{2}\left[\frac{\ln \left(R / R_{\mathrm{m}}\right)}{s}\right]^{2}\right\} .
$$

When the dispersion parameter $s$ is sufficiently small, $s R_{\mathrm{m}}$ is a good approximation for the standard deviation of the distribution and $R_{\mathrm{m}}$ is a very good approximation for the average radius of the distribution $\bar{R}$, as defined by

$$
\bar{R}=\frac{\int_{0}^{\infty} R f(R) \mathrm{d} R}{\int_{0}^{\infty} f(R) \mathrm{d} R}=R_{\mathrm{m}} \exp \left(\frac{\sigma^{2}}{2}\right) \cong R_{\mathrm{m}} .
$$


The intensity scattered by a single spherical particle of size $R$ and volume $V$ at a scattering vector $q$ is well known to be equal to [see e.g. Guinier \& Fournet (1955) or Glatter \& Kratky (1982)]

$$
I_{\text {sphere }}(q, R)=K V^{2}\left[\frac{\sin (q R)-q R \cos (q R)}{(q R)^{3}}\right]^{2},
$$

where $K$ is proportional to the square of the contrast in electron density. In the dilute approximation, interparticle interference can be neglected, and thus the scattering due to a distribution of spheres can be easily calculated as

$$
I(q)=\int_{0}^{\infty} I_{\text {sphere }}(q, R) f(R) \mathrm{d} R .
$$

Figs. 1 and 2 show SAS curves for lognormal PSDs of spheres, changing the dispersion parameter $s$, in the Guinier and

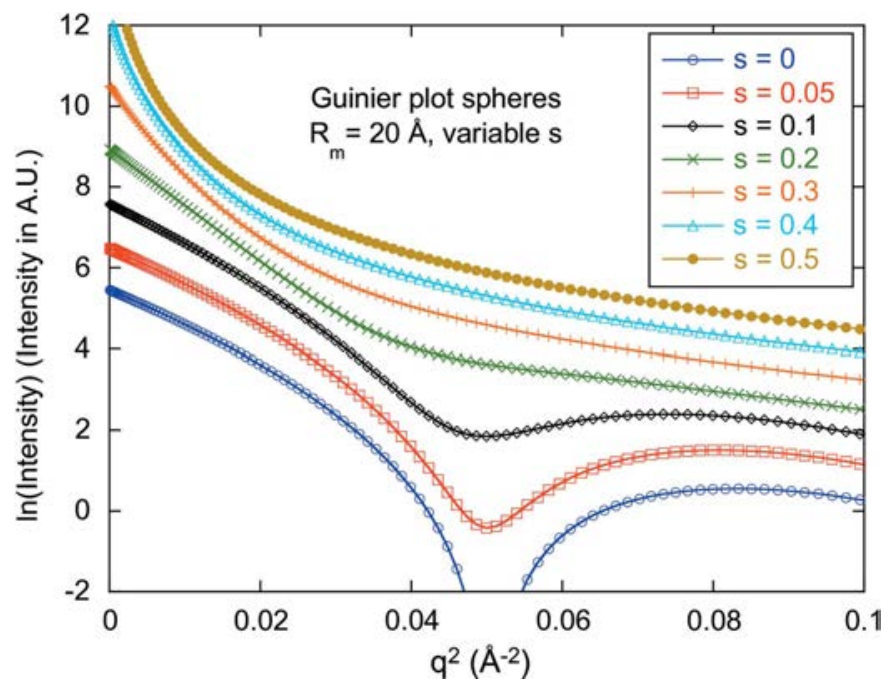

Figure 1

Scattered intensity calculated for lognormal distributions of spheres with $R_{\mathrm{m}}=20 \AA$ and variable $s$ in a Guinier representation.

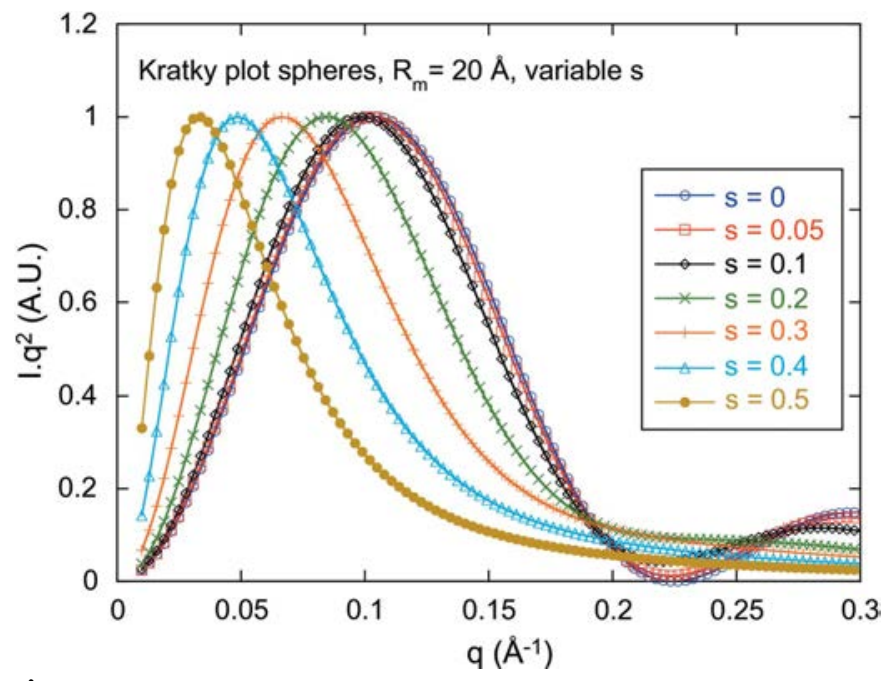

Figure 2

Scattered intensity calculated for lognormal distributions of spheres with $R_{\mathrm{m}}=20 \AA$ and variable $s$ in a Kratky representation.
Kratky representations, respectively, using an $R_{\mathrm{m}}$ value of $20 \AA$. For clarity, in the Guinier representation all the curves have been shifted by a constant value (corresponding to a multiplication factor), while the Kratky plots have been normalized by the maximum value of $I q^{2}$.

For the Guinier representation, in the monodisperse case no extended linear region appears in the plot; the theoretical radius corresponding to equation (2) is found in the low- $q$ range. The $q$ range where this estimate can be made is restricted to about $q R=1$ (i.e. $q=0.05$ here). At larger scattering vectors the behaviour deviates very quickly from the linear Guinier approximation. This can be best observed by representing the local apparent Guinier radius, which, for a given scattering vector $q$, is

$$
R_{\mathrm{g}}^{\mathrm{ap}}(q)=[-3 \alpha(q)]^{1 / 2},
$$

where $\alpha(q)$ is the local derivative of $\ln (I)$ versus $q^{2}$.

Fig. 3 shows such a plot for different values of $s$. When the dispersion increases, the Guinier plot progressively changes towards a less convex curve. For $s=0.2$ (corresponding approximately to a relative standard deviation of $20 \%$ in the PSD), the Guinier plot is actually observed to be very linear up to relatively large values of $q$. Calculating the Guinier radius from the slope of the straight line of the plot leads to a value very close to $20 \AA$, which is precisely that of the average precipitate size and quite different from the value given by equation (2). The validity range of the linear slope extends to approximately $q R=2.5$. As discussed above, this $q$ range corresponds to that used in several papers measuring precipitate sizes with the Guinier approximation. For $s$ values equal to or larger than 0.3 , the SAS curves loose their linear character and become markedly concave.

In the Kratky representation (Fig. 2), it is observed that all the SAS curves present a well defined maximum, which can be analysed in terms of a pseudo-Guinier radius. However, although for all calculations the average radius was the same,

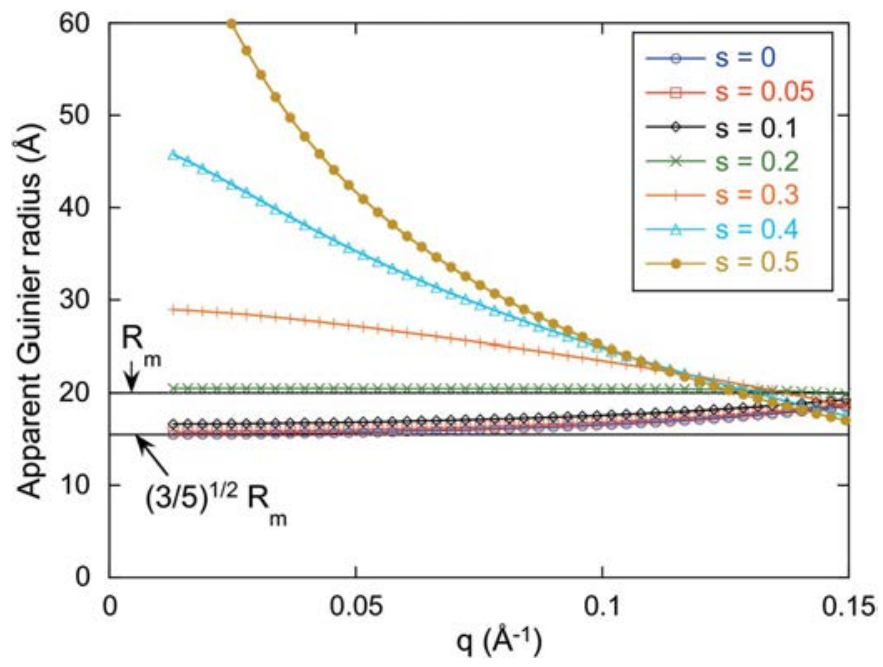

Figure 3

Apparent Guinier radius, plotted as a function of scattering vector $q$ for lognormal distributions of spheres with $R_{\mathrm{m}}=20 \AA$ and variable $s$. 
the measured pseudo-Guinier radius depends strongly on the width of the PSD. It is interesting to normalize the $q$ axis of the Kratky representations by the scattering vector where they show a maximum. This is done in Fig. 4, and shows that when the dispersion increases the normalized width of the scattering curve increases. Therefore, this normalized width could be used for a rapid estimation of the width of the precipitate size distribution. The absolute width of the Kratly plot peak, on the other hand, is shown to decrease when the dispersion increases, due to a stronger decrease in $q_{\max }$.

A systematic study of Guinier and pseudo-Guinier radii variations as a function of the width of the PSD will now be presented. However, it is first necessary to devise a procedure for measuring the Guinier radius in a self-consistent way. In fact, self-consistency imposes that the boundaries of the interval where the slope of the Guinier curve is measured must be constant values of $q R_{\mathrm{g}}$. A starting estimate of $R_{\mathrm{g}}$ is thus needed. The procedure that we apply is the following. First, the pseudo-Guinier radius $\left(R_{\max }\right)$ is calculated by measuring precisely the location of the maximum of the Kratky plot and using equation (4). This $R_{\max }$ value is taken as a first estimate for the boundaries of the linear fit in the Guiner plot, which are taken as $A / R_{\max }$ and $B / R_{\max } . A$ and $B$ are thus the parameters defining the extension of the Guinier fit. The Guinier fit between these boundaries leads to a first value of the Guinier radius, which is used to refine the fit boundaries. This procedure is repeated until convergence is reached, and leads to a final value of the Guinier radius. The procedure enables the calculation of a self-consistent value of the Guinier radius, irrespective of the actual precipitate size or size distribution. In the following, we will use the values $A=1$ and $B=2$ for the calculations.

In addition to the Guinier and pseudo-Guinier radii, we also measured the Porod radius, which represents the radius of a sphere of identical surface-to-volume ratio as the measured

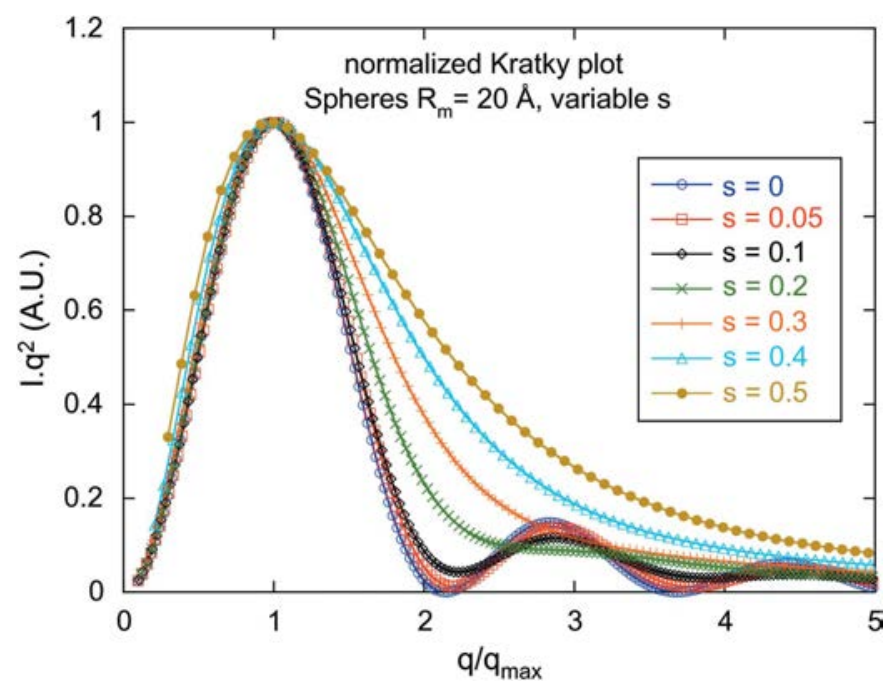

Figure 4

Scattered intensity calculated for lognormal distributions of spheres with $R_{\mathrm{m}}=20 \AA$ and variable $s$ in a Kratky representation, where the scattering vectors of all curves have been normalized by the value at which $I q^{2}$ shows a maximum. precipitate size distribution (Glatter \& Kratky, 1982; Kostorz, 1996):

$$
R_{\mathrm{p}}=3 V / S
$$

where $V$ is the total volume of the precipitates and $S$ the total surface of their interface with the matrix.

This radius is calculated by first estimating the asymptotic behaviour at large scattering vectors,

$$
I(q) \rightarrow I_{0}+K_{\mathrm{p}} / q^{4},
$$

where $I_{0}$ is a constant that represents the background noise of the measurement. In practice it contains terms related to the Laue scattering of the disordered solid solution, possible fluorescence of secondary chemical species present in the material and possible defaults in the estimation of the ground level for the detector. In our numerical calculations, $I_{0}=0$.

The estimation of the Porod radius is derived from the measured value of $K_{\mathrm{p}}$ and also requires the value of the integrated intensity of the scattering signal, namely the area below the Kratky plot, extrapolated to infinite scattering vector using the Porod asymptotic behaviour (such as used by Dumont et al., 2005):

$$
\begin{gathered}
Q_{0}=\int_{0}^{\infty} I q^{2} \mathrm{~d} q \\
R_{\mathrm{p}}=\frac{3 Q_{0}}{\pi K_{\mathrm{p}}} .
\end{gathered}
$$

It must be stressed that the measurement of the Porod radius from experimental data is subject to much larger uncertainties than the measurements of the Guinier and pseudo-Guinier radii, since both $Q_{0}$ and $K_{\mathrm{p}}$ are difficult to determine with high precision.

Fig. 5 shows the values of $R_{\mathrm{g}}, R_{\max }$ and $R_{\mathrm{p}}$ measured by this procedure on the SAS patterns calculated from lognormal

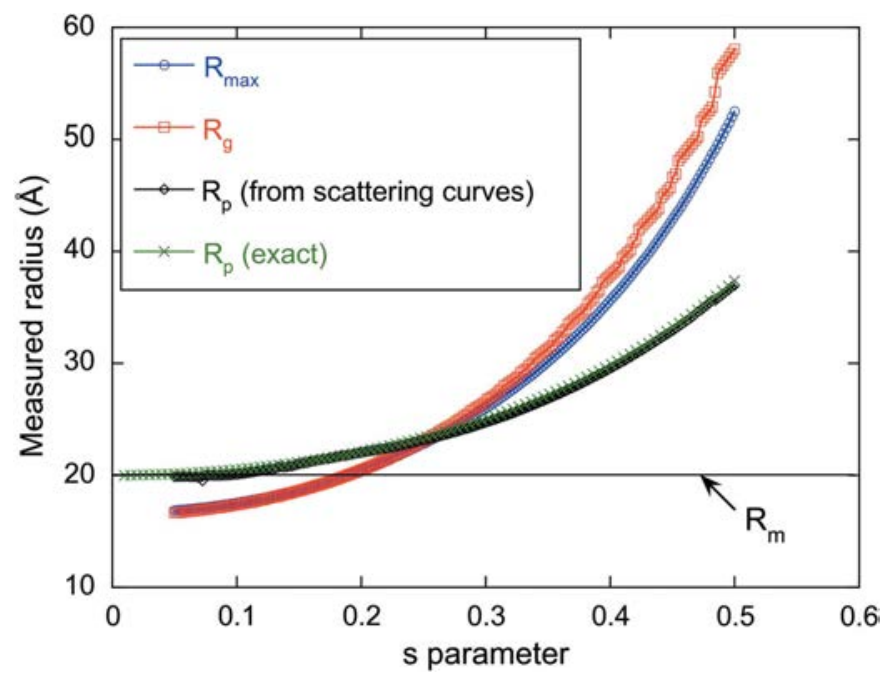

Figure 5

Values of pseudo-Guinier radius $\left(R_{\max }\right)$, Guinier radius $\left(R_{\mathrm{g}}\right)$ and Porod radius $\left(R_{\mathrm{p}}\right)$, measured using the procedure described in the text from scattering curves calculated from lognormal distributions of spheres with $R_{\mathrm{m}}=20 \AA$ and variable $s$. The exact value of $R_{\mathrm{p}}$ for these distributions, calculated from the volume-to-surface ratio, is also indicated. 
distributions with $R_{\mathrm{m}}=20 \AA$ and variable $s$. In agreement with Fig. 1, the measured radius increases monotonically with the width of the distribution. For very narrow distributions, the value of $(3 / 5)^{1 / 2} R$ is approached. However, it is not reached because the chosen values of $A$ and $B$ do not satisfy the $q R<<1$ condition. For an $s$ value of approximately 0.2 , the Guinier radius is shown to be equal to the average radius of the PSD. Above a PSD width of 0.3 , a large deviation is found between the measured Guinier radius and the average precipitate size. With this set of $A$ and $B$ parameters, the Guinier and pseudoGuinier measurements give very consistent results, even when the $\ln (I)$ versus $q^{2}$ curve is not straight. Consequently, comparing both values may provide information on the validity of the underlying hypotheses made here (spherical particles, lognormal distribution).

The Porod radius is observed to increase when the dispersion increases. For a dispersion parameter below 0.25, the Porod radius is significantly larger than the Guinier radius, whereas the contrary is observed above. A comparison between the Guinier and Porod radii is therefore a valuable indication of the width of the precipitate size distribution in the case of spheres. Furthermore, it is useful to check the agreement of the Porod radius measurement from the calculated scattering curves with the exact value calculated from the precipitate size distribution:

$$
R_{\mathrm{p}}=\frac{\int_{0}^{\infty} R^{3} f(R) \mathrm{d} R}{\int_{0}^{\infty} R^{2} f(R) \mathrm{d} R} .
$$

Fig. 5 shows that the agreement between the measured and calculated Porod radii is excellent, which validates this procedure for radius estimation.

\section{SAS from a lognormal distribution of ellipsoids}

Similar calculations will now be performed for ellipsoids of revolution. We will investigate both the oblate case $(a=b>c$, where $a, b$ and $c$ are the three semi-axes), which corresponds to platelet-like ellipsoids, and the prolate case $(a=b<c)$, which corresponds to needle-like ellipsoids. The ellipsoids will be assumed to be distributed in terms of size according to a lognormal distribution and to have a constant aspect ratio $(k=c / a)$.

The scattering behaviour of a single ellipsoid obeys the same function as that of a virtual sphere, for which the radius $R_{\text {eq }}$ depends on the angles $(\alpha, \beta, \gamma)$ between the three axes of the ellipsoid and the scattering vector (Glatter \& Kratky, 1982):

$$
R_{\mathrm{eq}}^{2}=a^{2} \cos ^{2} \alpha+b^{2} \cos ^{2} \beta+c^{2} \cos ^{2} \gamma .
$$

Since two of the axes are equal, it is possible to set $\beta=\pi / 2$ and $\alpha=\gamma-\pi / 2$. Then,

$$
R_{\mathrm{eq}}^{2}=a\left[1+\cos ^{2} \gamma\left(k^{2}-1\right)\right]^{1 / 2} .
$$

It is possible to express this as a function of the radius of a sphere of equivalent volume $R_{\text {ell }}$, which we will use in the following as the main parameter to describe the precipitate size distribution:

$$
\begin{gathered}
R_{\mathrm{ell}}=a k^{1 / 3}=c / k^{2 / 3}, \\
R_{\mathrm{eq}}\left(R_{\mathrm{ell}}, \gamma\right)=\frac{R_{\mathrm{ell}}}{k^{1 / 3}}\left[1+\cos ^{2} \gamma\left(k^{2}-1\right)\right]^{1 / 2} .
\end{gathered}
$$

It follows that, in order to obtain the intensity scattered by a distribution of ellipsoids under the assumption that their orientations are randomly distributed (no texture effect), one has simply to integrate first on the orientation of the ellipsoid and then on the precipitate size distribution:

$$
\begin{gathered}
I_{\text {ell }}\left(q, R_{\text {ell }}\right)=\int_{0}^{\pi / 2} I_{\text {sphere }}\left[q, R_{\text {eq }}\left(R_{\text {ell }}, \gamma\right)\right] \sin \gamma \mathrm{d} \gamma, \\
I(q)=\int_{0}^{\infty} I_{\text {ell }}(q, R) f(R) \mathrm{d} R .
\end{gathered}
$$

Now we will evaluate, similarly to the case of spheres, the effect of the aspect ratio of the particles on the Guinier, Kratky and Porod behaviours of the scattered intensity. In order to minimize the number of varying parameters, we will keep the dispersion parameter of the lognormal distribution at a constant value of 0.2 . The average radius $R_{\mathrm{m}}$ of the precipitate size distribution (which is here the radius of a sphere of equivalent volume to the ellipsoid) is also kept at a constant value of $20 \AA$.

Figs. 6 and 7 show the calculated scattering intensities for different values of the aspect ratio in the Guinier and Kratky representations, respectively. The first feature is that the effect of changing the aspect ratio from that of a sphere is qualitatively similar whether it is increased or decreased. This means that it is necessarily difficult to distinguish, from scattering curves alone, a distribution of needle-like ellipsoids from one

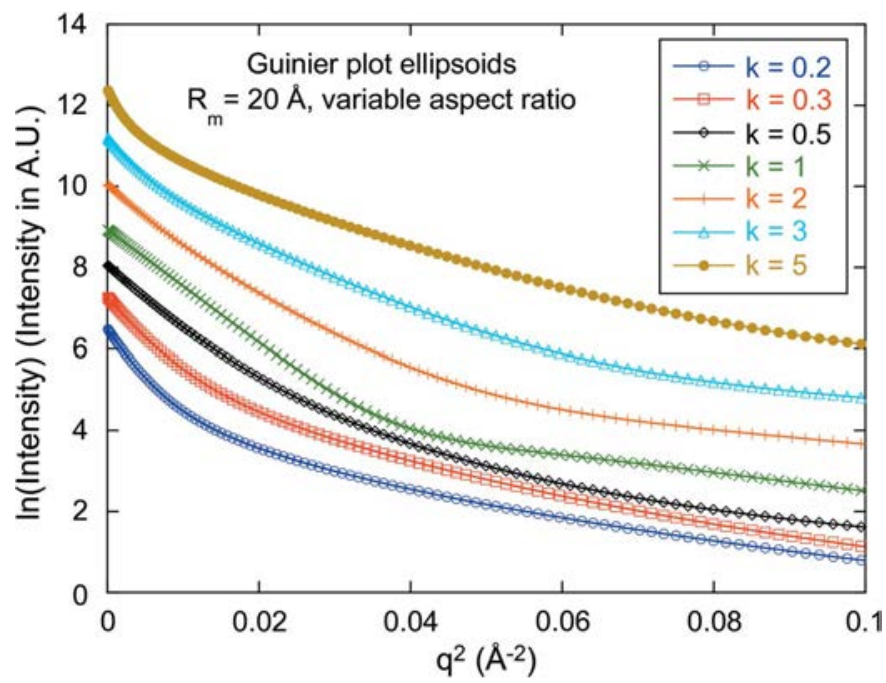

Figure 6

Scattered intensity calculated for lognormal distributions of ellipsoids with $R_{\mathrm{m}}=20 \AA$ and variable aspect ratio, $s$ being kept constant at 0.2 , in a Guinier representation. 
of plate-like ellipsoids. In more detail, it is observed that having an aspect ratio different from 1 disrupts the straight line in the Guinier plot that is observed for the distribution of spheres in the case when $s=0.2$. However, this disruption is moderate if the aspect ratio is between 0.5 and 2 . On the Kratky representation, the scattering at small angles (before the maximum of $\left.I q^{2}\right)$ is little affected by the value of the aspect ratio, but the scattering at wider angles increases dramatically when the aspect ratio becomes very different from 1. Again, little modification is observed when the aspect ratio stays in the range $0.5-2$.

This is further confirmed (see Fig. 8) by the evaluation of the different parameters that can be extracted from the scattering curves (pseudo-Guinier radius, Guinier radius, Porod radius; see §2). If the aspect ratio stays in the range $0.5-2$, the Guinier and pseudo-Guinier radii stay close to the average radius of the PSD. For more pronounced aspect ratios, these radii depart moderately from this value (within a $25 \%$ precision for the range of aspect ratios investigated). The Porod radius is also logically observed to decrease when pronounced aspect ratios are used, in relation to the increase in the developed surface of the ellipsoids. The exact Porod radius can also be calculated in the present case:

$$
R_{\mathrm{p}}=\frac{\int_{0}^{\infty} V_{\mathrm{ell}}(R) f(R) \mathrm{d} R}{\int_{0}^{\infty} S_{\mathrm{ell}}(R) f(R) \mathrm{d} R},
$$

with

$$
\begin{gathered}
V_{\mathrm{ell}}(R)=\frac{4}{3} \pi R^{3}, \\
\text { Oblate } S_{\text {ell }}(R)=2 \pi\left[a^{2}+c^{2} \frac{\tanh ^{-1}(\sin \varepsilon)}{\sin \varepsilon}\right],
\end{gathered}
$$

with $\varepsilon=\cos ^{-1}(k)$, and

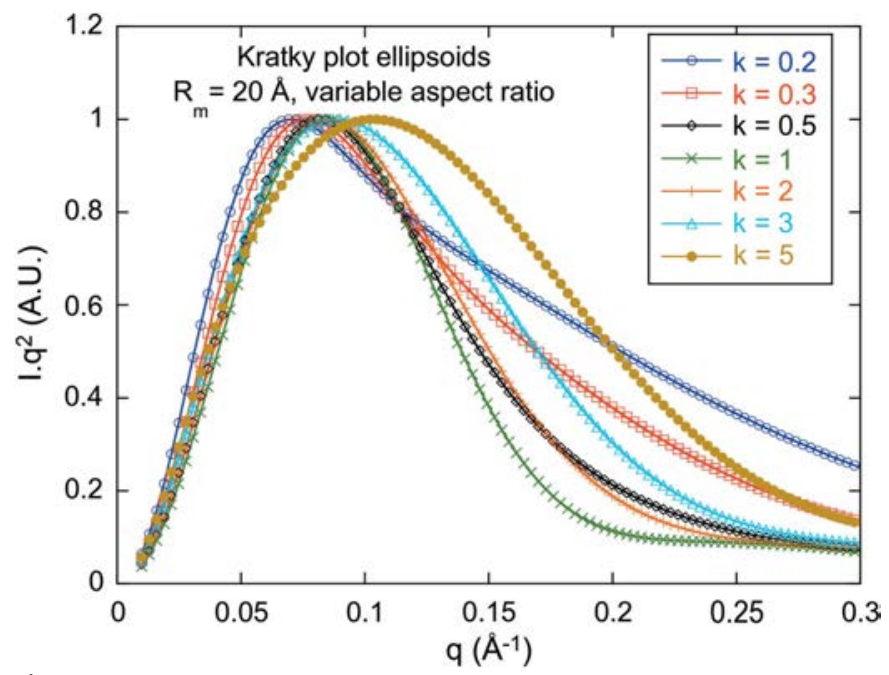

Figure 7

Scattered intensity calculated for lognormal distributions of ellipsoids with $R_{\mathrm{m}}=20 \AA$ and variable aspect ratio, $s$ being kept constant at 0.2 , in a Kratky representation.

$$
\text { Prolate } S_{\text {ell }}(R)=2 \pi\left(a^{2}+c^{2} \frac{\varepsilon}{\tan \varepsilon}\right),
$$

with $\varepsilon=\cos ^{-1}(1 / k)$, where $a$ and $c$ are calculated from $R$ using equation (17). Fig. 8 shows good agreement between this exact calculation of the Porod radius and that measured from the calculated scattering curves, except for very large (or very small) aspect ratios where a small deviation is observed. This comes from the fact that the scattering curves corresponding to these cases extend to very large scattering vectors, which hinders precise evaluation of the asymptotic $1 / q^{4}$ Porod.

\section{Application to experimental data in the case of spherical particles: the Fe-Cu system}

The $\mathrm{Fe}-\mathrm{Cu}$ system is a classical prototype system for the study of precipitation (Othen et al., 1991; Osamura et al., 1993; Deschamps et al., 2001; Perez et al., 2005). It is known that, in the first stages of precipitation, the particles are spherical to a good approximation, and furthermore the volume fraction is low enough to prevent interparticle effects $\left(f_{\mathrm{v}} \simeq 1 \%\right)$ but still sufficiently high to enable high-precision measurements. In this system, scattering can be recorded using either X-rays or neutrons, although the former are preferable for in situ measurements during heat treatments. Measurements in this case need to be performed using synchrotron radiation with a wavelength close to the $K \alpha$-edge of $\mathrm{Fe}$, in order to increase the weak contrast between $\mathrm{Cu}$ and $\mathrm{Fe}$ atoms. The results shown in this section were obtained on a binary $\mathrm{Fe}-1.4 \mathrm{wt} \%$ alloy that was solutionized for $5 \mathrm{~h}$ at $1123 \mathrm{~K}$ in a sealed tube and subsequently quenched in cold water prior to ageing. The grain size was between 50 and $100 \mu \mathrm{m}$. The sample was then thinned mechanically to a thickness of $100 \mu \mathrm{m}$ and electrochemically down to $50 \mu \mathrm{m}$ prior to the in situ heating carried

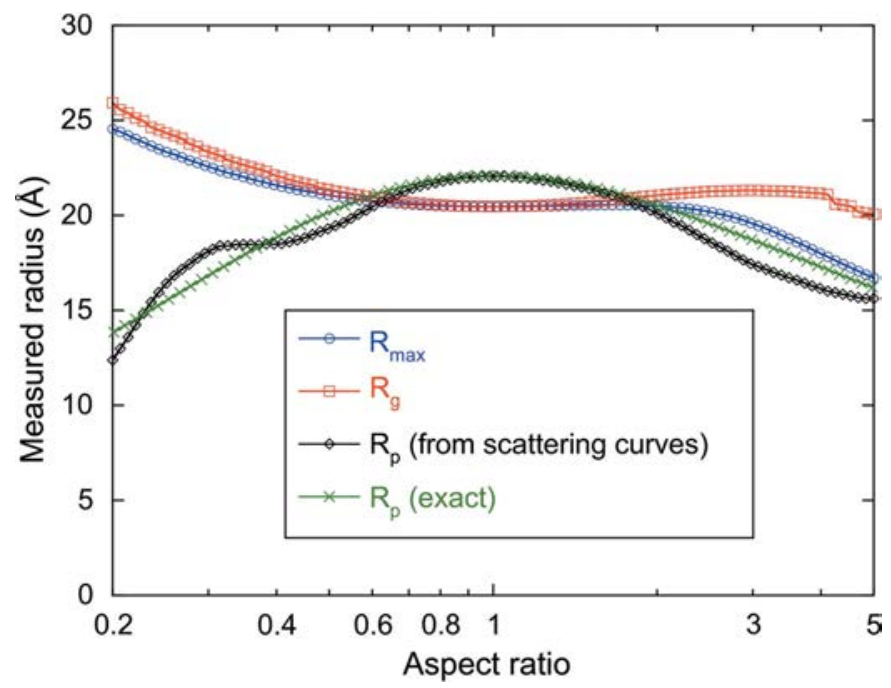

Figure 8

Values of pseudo-Guinier radius $\left(R_{\max }\right)$, Guinier radius $\left(R_{\mathrm{g}}\right)$ and Porod radius $\left(R_{\mathrm{p}}\right)$, measured using the procedure described in the text from scattering curves calculated from lognormal distributions of ellipsoids with variable aspect ratio. The exact value of $R_{\mathrm{p}}$ for these distributions, calculated from the volume-to-surface ratio, is also indicated. 


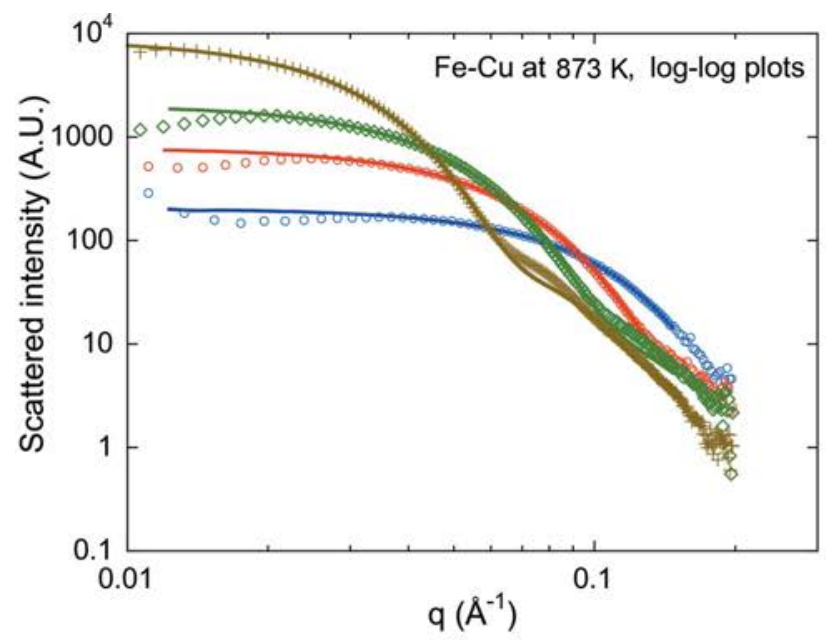

(a)

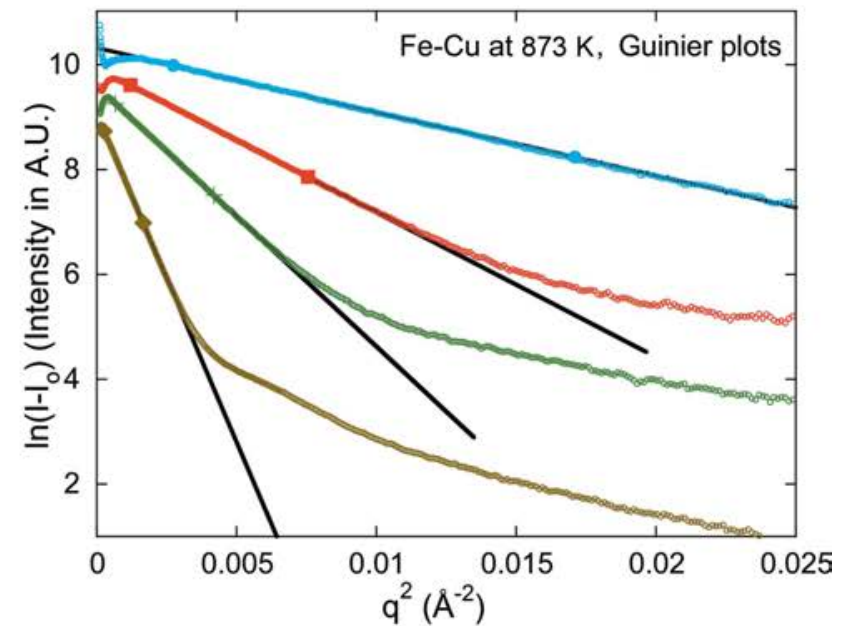

(b)

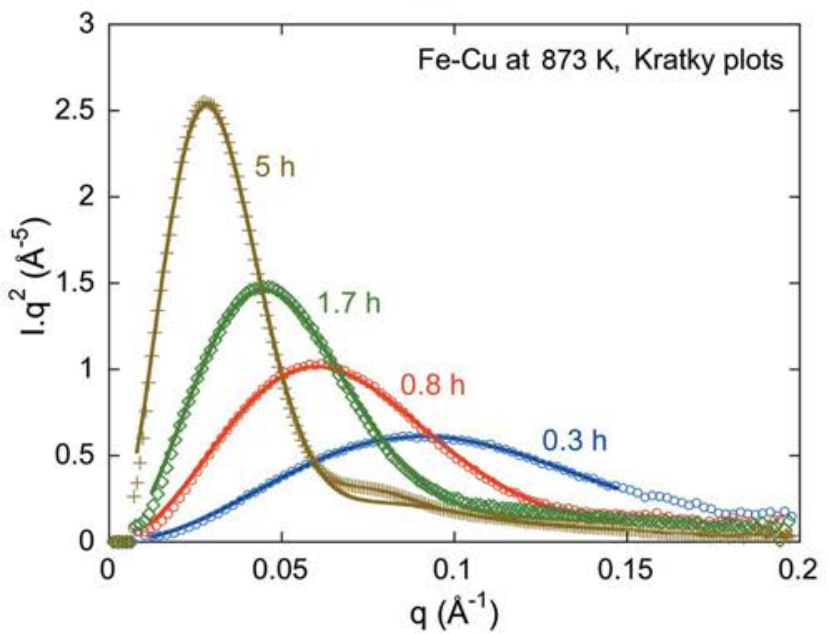

(c)

Figure 9

Scattering curves for four selected ageing times $(0.3,0.8,1.7$ and $3 \mathrm{~h})$ during heat treatment at $873 \mathrm{~K}$ on the $\mathrm{Fe}-\mathrm{Cu}$ alloy. (a) Raw scattering curves (symbols) and model fitting curves (solid lines). (b) Guinier plot. The fitted straight lines were determined within the boundaries in a selfconsistent way (see $\$ 2$ ). (c) Kratky plot. The average radius $R_{\mathrm{m}}$ and the polydispersity parameter were determined from the model fitting curves (solid lines). out under the X-ray beam. Experiments were carried out at the D2AM/BM02 beamline of the European Synchrotron Research Facility (ESRF) in Grenoble, France, with an X-ray energy of $7104 \mathrm{eV}$. The beam size was approximately $100 \times 200 \mu \mathrm{m}$. Scattered intensity was recorded by a CCD camera and corrected for read-out noise, flat field and grid distortion, and background noise. We will present here measurements made in situ at $873 \mathrm{~K}$ during the SAXS recording, starting from a homogeneous solid solution.

Figs. $9(a), 9(b)$ and $9(c)$ show four selected scattering curves in $\log -\log$, Guinier and Kratky representations, respectively. It can be seen in the Guinier plots (Fig. 9b) that the scattering curves are remarkably linear up to large values of the scattering vector, which was shown in $\$ 2$ to be characteristic of a dispersion parameter of about 0.2. In the Kratky plot (Fig. 9c), calculated scattering curves are also represented, for which the two parameters of a lognormal distribution $\left(R_{\mathrm{m}}\right.$ and $\left.s\right)$ have been adjusted to the data. The fit is excellent, which further validates the use of lognormal distributions to model the data.

Now the whole set of data can be evaluated using the procedure described above, namely the Guinier, pseudoGuinier and Porod radii, and also the $R_{\mathrm{m}}$ and $s$ parameters of the PSD, by fitting of the whole scattering curve.

The results of this analysis are shown in Fig. 10. This demonstrates that the procedure described for calculating the Guinier and pseudo-Guinier radii leads to a very high reproducibility, since almost no noise is present in the resulting data. The Guinier and pseudo-Guinier radii are indistinguishable, which is expected from a lognormal distribution of spheres with a dispersion of the order of 0.2 . The average radius $R_{\mathrm{m}}$ resulting from the fit diverges by only about $5 \%$ from the above two parameters, which is also consistent with the calculations made in $\S 2$. The $s$ parameter is shown to be relatively constant throughout the whole heat treatment process at values fluctuating between 0.22 and 0.2 , showing that the coarsening stage of precipitates occurs in a self-similar fashion. Finally, the Porod radius, although noisier, shows

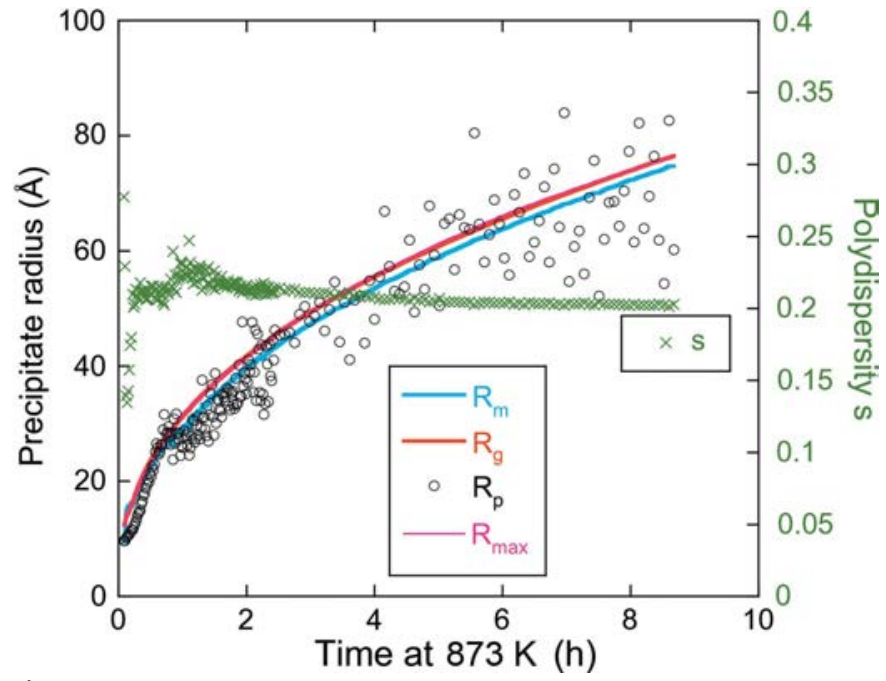

Figure 10

Parameters extracted from the scattering curves through the whole $873 \mathrm{~K}$ heat treatment on the $\mathrm{Fe}-\mathrm{Cu}$ alloy. 
values equivalent to the other radii. From Fig. 5, it is expected that this should happen for a dispersion of 0.25 , which is close to that found here.

It can be concluded from these results that the lognormal distribution is a good approximation of the experimental PSD, that 0.2 is a typical dispersion value during conventional heat treatments, and that under these conditions the Guinier radius (or pseudo-Guinier radius) is a very good estimate of the average precipitate size.

\section{Application to experimental data in the case of ellipsoidal particles: the $\mathrm{Fe}-\mathrm{Nb}-\mathrm{C}$ system}

In $\mathrm{Fe}, \mathrm{NbC}$ precipitates as platelet-like particles, nucleated on dislocations. Due to the low volume fraction (usually lower than $0.1 \%$ ), and the poor X-ray contrast between precipitate and matrix, this system is best studied using neutron scattering. The non-magnetic nature of the precipitate makes it possible to perform the measurement under a saturating magnetic field and extract from the data the magnetic scattering contribution resulting only from the 'magnetic holes' present in the Fe matrix (Kostorz, 1996). The alloy, with a composition (in atomic p.p.m.) of $\mathrm{Fe}-470 \mathrm{Nb}-510 \mathrm{C}$, was solutionized in a sealed quartz tube at $1513 \mathrm{~K}$ for $45 \mathrm{~min}$ in the austenitic state and then quenched in cold water, resulting in a quasipolygonal fully ferritic microstructure with a small grain size (approximately $3 \mu \mathrm{m}$ ) and a relatively large dislocation density. The material was then aged for $300 \mathrm{~min}$ at $973 \mathrm{~K}$. Samples were subsequently prepared to $3 \mathrm{~mm}$ thickness, mirror-polished mechanically and slightly electropolished. Measurements were carried out on the D22 beamline of the Institut Laue-Langevin (ILL) in Grenoble, France, at a neutron wavelength of $6 \AA$. Three distances were used to obtain the full $q$ range presented here. The data were analysed using the beamline software GRASP (http://www.ill.eu/lss/ grasp).

Representative scattering curves (in log-log, Guinier and Kratky representations, respectively) are shown in Figs. 11(a), $11(b)$ and 11(c). Fig. 11(b) shows that, as expected, no linear Guinier behaviour is observed. However, the self-consistent method for determining the Guinier radius, as detailed in $\S 2$, can be used to obtain a value for $R_{\mathrm{g}}$ of $33.6 \AA$. The Kratky plot shows a well defined peak, which can be used to calculate a pseudo-Guinier radius of $30.7 \AA$. This radius is somewhat smaller than the Guinier radius, which is consistent with the calculations of $\S 3$ (see Fig. 8). The Porod radius can be computed as well, and one finds a value of $21 \AA$, which is much smaller than the pseudo-Guinier radius. Again, this is consistent with the calculations presented in Fig. 8. From Fig. 8, the precipitate aspect ratio, corresponding to the experimental ratio between the pseudo-Guinier and Porod radii, can be estimated to be 0.25 . This value is relatively close to that obtained by fitting the scattering curve with a distribution of ellipsoids, namely 0.32 (note that, owing to parasitic scattering of large objects at very small angles, it was only possible to fit the data above $q=0.045 \AA^{-1}$ ). Also, this fit gives an average radius $R_{\mathrm{m}}$ of $25.4 \AA$, which is smaller than the Guinier radius, as predicted by the calculations of Fig. 8 .
The conclusion of this evaluation is that, although more caution should be exercised when evaluating microstructures consisting of ellipsoidal precipitates as opposed to spherical

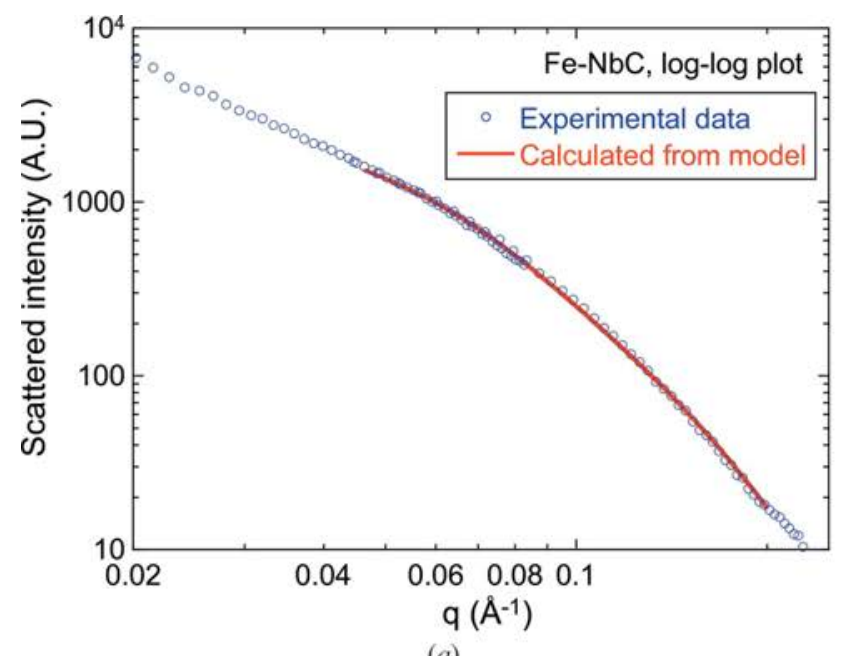

(a)

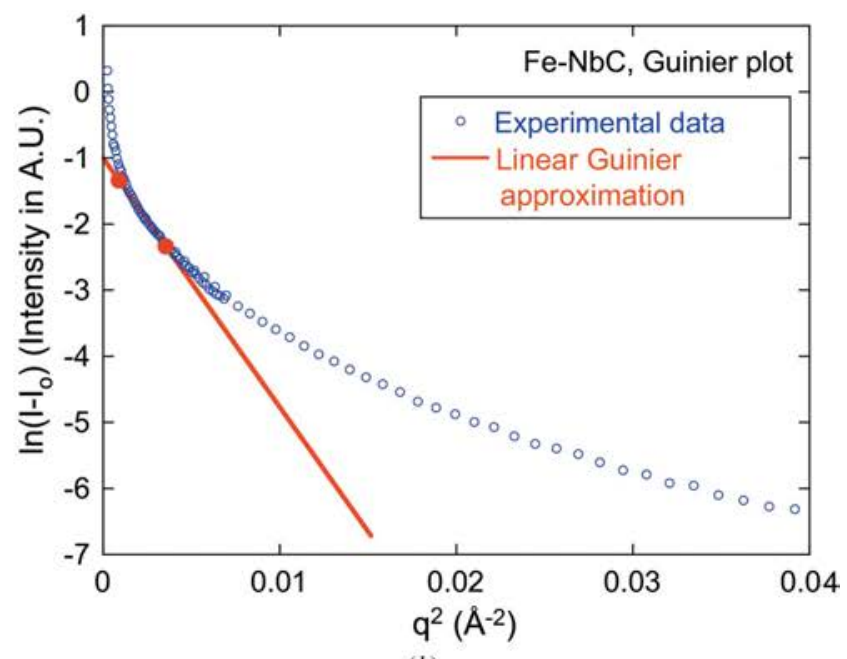

(b)

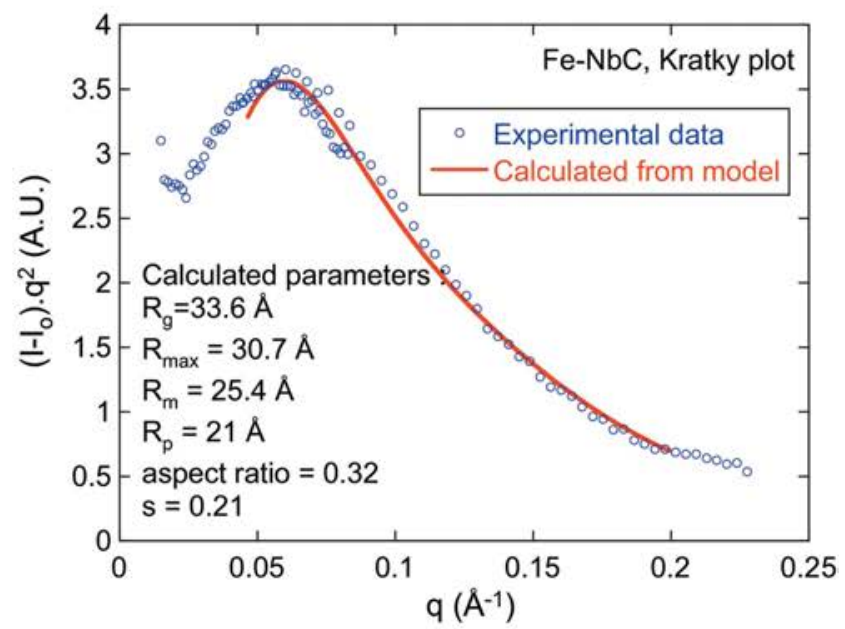

(c)

Figure 11

Magnetic scattering data for the Fe-Nb-C alloy. (a) Raw scattering curve, along with a fit for a model containing plate-like ellipsoidal precipitates. (b) Guinier plot. (c) Kratky plot and the calculated parameters of the microstructure. 
precipitates, the comparison between the different parameters of the microstructure that can be obtained by simple measurements $\left(R_{\mathrm{g}}, R_{\mathrm{m}}, R_{\mathrm{max}}, R_{\mathrm{p}}\right)$ provides good estimates of the precipitate size and morphology.

\section{Conclusions}

The calculations performed in this paper make it possible to assess the confidence in the measurement of precipitate sizes using simple and straightforward methods, such as the evaluation of the Guinier radius, the pseudo-Guinier radius and the Porod radius. These methods are particularly suitable when a large amount of data needs to be evaluated.

We have shown that, for a polydisperse collection of spherical particles following a lognormal size distribution, the Guinier regime extends to relatively large values of $q$ when the dispersion parameter is about $20 \%$. We have shown that this is a situation often encountered in practice. In addition, we have proposed a self-consistent method of determining the Guinier radius to overcome the subjective choice of fit boundaries.

We have demonstrated that, when the precipitates have a moderate aspect ratio and a dispersion of about $20 \%$, the measurement of the Guinier radius provides a precise estimate of the average precipitate size. In such cases, the correction factor that applies to the case of a monodisperse collection of spheres should not be used, in contrast with what is sometimes done in the literature. It has also been shown that simultaneously measuring the 'pseudo-Guinier' radius from the Kratky plot provides an estimate of the confidence that one can have in these measurements. In cases where the Guinier and pseudo-Guinier radii deviate strongly, precipitates can be predicted to have either a pronounced aspect ratio or a wider precipitate size distribution. Furthermore, a comparison between these radii and the Porod radius provides useful estimates of the width of the precipitate size distribution or of the precipitate aspect ratio.

When dealing with ellipsoid-shaped particles, we have shown that the relationships existing between the abovementioned measured precipitate sizes and the average size of the distribution are almost not affected for aspect ratios in the range $0.5-2$. For larger aspect ratios strong differences occur, and one should use the Guinier radius, with caution, simply to describe the average precipitate size. In such cases a full model fitting is certainly indicated.

The authors acknowledge the help of Dr Françoise Bley and the staff at the BM02/D2AM beamline (ESRF, Grenoble) during the SAXS experiments and data processing, and of Dr Charles Dewhurst (ILL, Grenoble) for the SANS experiments and interpretation. Dr Fabien Perrard is also acknowledged for the $\mathrm{Fe}-\mathrm{Nb}-\mathrm{C}$ data.

\section{References}

Alinger, M. J., Odette, G. R. \& Hoelzer, D. T. (2009). Acta Mater. 57, 392-406.

Antion, C., Bley, F., Donnadieu, P., Deschamps, A., Pisch, A., Blandin, J.-J. \& Tassin-Arques, C. (2007). J. Appl. Cryst. 40, s126-s131. Bergner, F., Ulbricht, A. \& Heintze, C. (2009). Scr. Mater. 61, 1060-1063.
Bischof, M., Erlach, S., Staron, P., Leitner, H., Scheu, C. \& Clemens, H. (2005). Z. Metallkd. 96, 1074-1080.

Bischof, M., Staron, P., Caliskanoglu, D., Leitner, H., Scheu, C. \& Clemens, H. (2008). Mater. Sci. Eng. Sect. A, 472, 148-156.

De Geuser, F., Bley, F., Denquin, A. \& Deschamps, A. (2010). J. Phys. Conf. Ser. 247, 012034.

Del Genovese, D., Rösler, J., Strunz, P., Mukherji, D. \& Gilles, R. (2005). Metall. Mater. Trans. A, 36, 3439-3450.

Deschamps, A., Genevois, C., Nicolas, M., Perrard, F. \& Bley, F. (2005). Philos. Mag. 85, 3091-3112.

Deschamps, A., Militzer, M. \& Poole, W. J. (2001). Iron Steel Inst. Jpn Int. 41, 196-205.

Dumont, M., Lefebvre, W., Doisneau-Cottignies, B. \& Deschamps, A. (2005). Acta Mater. 53, 2881-2892.

Dumont, M., Steuwer, A., Deschamps, A., Peel, M. \& Withers, P. J. (2006). Acta Mater. 54, 4793-4801.

Dupasquier, A., Ferragut, R., Iglesias, M. M., Massazza, M., Riontino, G., Mengucci, P., Barucca, G., Macchi, C. E. \& Somoza, A. (2007). Philos. Mag. 87, 3297-3323.

Glade, S. C., Wirth, B. D., Odette, G. R., Asoka-Kumar, P., Sterne, P. A. \& Howell, R. H. (2005). Philos. Mag. 85, 629-639.

Glatter, O. (1977). J. Appl. Cryst. 10, 415-421.

Glatter, O. (1980). J. Appl. Cryst. 13, 7-11.

Glatter, O. \& Kratky, O. (1982). Small-Angle X-ray Scattering. London: Academic Press.

Guinier, A. \& Fournet, G. (1955). Small-Angle Scattering of X-rays. New York: John Wiley and Sons.

Guinier, A., Fournet, G., Walker, C. B. \& Vineyard, G. H. (1956). Phys. Today, 9, 38.

Kamiyama, T., Kimura, H., Sasamori, K. \& Inoue, A. (2001). Scr. Mater. 44, 1297-1301.

Kenesei, P., Horvath, G., Bernstorff, S., Ungar, T. \& Lendvai, J. (2006). Z. Metallkd. 97, 315-320.

Kompatscher, M., Deme, B., Kostorz, G., Somsen, C. \& Wassermann, E. F. (2002). Acta Mater. 50, 1581-1586.

Kostorz, G. (1996). Phys. Metall. 4, 1116-1199.

Kusy, M., Riello, P. \& Battezzati, L. (2004). Acta Mater. 52, 5031-5041.

Leitner, H., Bischof, M., Clemens, H., Erlach, S., Sonderegger, B., Kozeschnik, E., Svoboda, J. \& Fischer, F. D. (2006). Adv. Eng. Mater. 8, 1066-1077.

Leitner, H., Clemens, H., Akre, J., Danoix, F. \& Staron, P. (2007). Int. J. Mater. Res. 98, 1093-1103.

Leitner, H., Staron, P., Clemens, H., Marsoner, S. \& Warbichler, P. (2005). Mater. Sci. Eng. Sect. A, 398, 323-331.

Marlaud, T., Deschamps, A., Bley, F. \& Baroux, B. (2008). Aluminium Alloys, Their Physical and Mechanical Properties, edited by J. Hirsch, B. Skrotzki \& G. Gottstein. Weinheim: Wiley-VCH.

Marlaud, T., Deschamps, A., Bley, F., Lefebvre, W. \& Baroux, B. (2010a). Acta Mater. 58, 248-260.

Marlaud, T., Deschamps, A., Bley, F., Lefebvre, W. \& Baroux, B. (2010b). Acta Mater. 58, 4814-4826.

Michaud, P., Delagnes, D., Lamesle, P., Mathon, M. H. \& Levaillant, C. (2007). Acta Mater. 55, 4877-4889.

Mukherji, D., Strunz, P., Del Genovese, D., Rösler, J., Gilles, R. \& Wiedenmann, A. (2003). Metall. Mater. Trans. A, 34, 2781-2792.

Ohnuma, M., Hono, K., Linderoth, S., Pedersen, J. S., Yoshizawa, Y. \& Onodera, H. (2000). Acta Mater. 48, 4783-4790.

Ohnuma, M., Suzuki, J., Ohtsuka, S., Kim, S. W., Kaito, T., Inoue, M. \& Kitazawa, H. (2009). Acta Mater. 57, 5571-5581.

Osamura, K., Okuda, H., Takashima, M., Asano, K. \& Furusaka, M. (1993). Mater. Trans. Jpn Inst. Met. 34, 305-311.

Othen, P. J., Jenkins, M. L., Smith, G. D. W. \& Phythian, W. J. (1991). Philos. Mag. Lett. 64, 383-391.

Perez, M., Perrard, F., Massardier, V., Kleber, X., Deschamps, A., De Monestrol, H., Pareige, P. \& Covarel, G. (2005). Philos. Mag. 85, 2197-2210.

Perrard, F., Deschamps, A., Bley, F., Donnadieu, P. \& Maugis, P. (2006). J. Appl. Cryst. 39, 473-482. 
Sato, S., Takahashi, Y., Sanada, T., Wagatsuma, K. \& Suzuki, S. (2009). J. Alloys Compd. 477, 846-850.

Schober, M., Lerchbacher, C., Eidenberger, E., Staron, P., Clemens, H. \& Leitner, H. (2010). Intermetallics, 18, 1553-1559.

Staron, P., Jamnig, B., Leitner, H., Ebner, R. \& Clemens, H. (2003). J. Appl. Cryst. 36, 415-419.

Staron, P. \& Kampmann, R. (2000). Acta Mater. 48, 701-712.

Tsao, C. S., Chen, C. Y., Jeng, U. S. \& Kuo, T. Y. (2006). Acta Mater. 54, 4621-4631.
Ulbricht, A., Bergner, F., Boehmert, J., Valo, M., Mathon, M. H. \& Heinemann, A. (2007). Philos. Mag. 87, 1855-1870.

Van Dijk, N. H., Bouwman, W. G., Offerman, S. E., Rekveldt, M. T., Sietsma, J., Van der Zwaag, S., Bodin, A. \& Heenan, R. K. (2002). Metall. Mater. Trans. A, 33, 1883-1891.

Vogel, M., Kraft, O., Staron, P., Clemens, H., Rauh, R. \& Arzt, E. (2003). Z. Metallkd. 94, 564-571.

Wiskel, J. B., Ivey, D. G. \& Henein, H. (2008). Metall. Mater. Trans. B, 39, 116-124. 